\title{
Evaluation of fluralaner and afoxolaner treatments to control flea populations, reduce pruritus and minimize dermatologic lesions in naturally infested dogs in private residences in west central Florida USA
}

Michael W. Dryden ${ }^{1 *}$, Michael S. Canfield ${ }^{2}$, Kimberly Kalosy ${ }^{1}$, Amber Smith ${ }^{1}$, Lisa Crevoiserat ${ }^{1}$, Jennifer C. McGrady ${ }^{1}$, Kaitlin M. Foley ${ }^{1}$, Kathryn Green ${ }^{2}$, Chantelle Tebaldi², Vicki Smith', Tashina Bennett ${ }^{1}$, Kathleen Heaney ${ }^{3}$, Lisa Math³ Christine Royal ${ }^{3}$ and Fangshi Sun ${ }^{3}$

\begin{abstract}
Background: A study was conducted to evaluate and compare the effectiveness of two different oral flea and tick products to control flea infestations, reduce pruritus and minimize dermatologic lesions over a 12 week period on naturally infested dogs in west central FL USA.

Methods: Thirty-four dogs with natural flea infestations living in 17 homes were treated once with a fluralaner chew on study day 0 . Another 27 dogs living in 17 different homes were treated orally with an afoxolaner chewable on day 0 , once between days 28-30 and once again between days 54-60. All products were administered according to label directions by study investigators. Flea populations on pets were assessed using visual area counts and premise flea infestations were assessed using intermittent-light flea traps on days $0,7,14,21$, and once between days 28-30, 40-45, 54-60 and 82-86. Dermatologic assessments were conducted on day 0 and once monthly. Pruritus assessments were conducted by owners throughout the study. No concurrent treatments for existing skin disease (antibiotics, anti-inflammatories, anti-fungals) were allowed.
\end{abstract}

Results: Following the first administration of fluralaner or afoxolaner, flea populations on pets were reduced by 99. $0 \%$ and $99.3 \%$, respectively within 7 days. Flea populations on the fluralaner treated dogs were 0 (100\% efficacy) on days 54-60 and 82-86 after the administration of a single dose on day 0. Administration of 3 monthly doses of afoxolaner reduced flea populations by $100 \%$ on days $82-86$. Flea numbers in indoor-premises were markedly reduced in both treatment groups by days $82-86$, with $100 \%$ and $98.9 \%$ reductions in flea trap counts in the fluralaner and afoxolaner treatment groups, respectively. Marked improvement was observed in FAD lesion scoring, Atopic Dermatitis lesions scoring (CADESI-4) and pruritus scores with both formulations.

Conclusions: In a clinical field investigation conducted during the summer of 2015 in subtropical Florida, a single administration of an oral fluralaner chew completely eliminated dog and premises flea infestations and markedly reduced dermatology lesions and pruritus. Three monthly doses of the afoxolaner chewable also eliminated flea infestations in dogs, markedly reduced premises' flea populations and similarly improved dermatology lesions and pruritus.

Keywords: Ctenocephalides felis felis, Cat flea, Dogs, Field study, Fluralaner, Flea, Flea control, Afoxolaner, Flea Allergy Dermatitis, Flea Bite Dermatitis, Pruritus, Atopic Dermatitis

\footnotetext{
* Correspondence: Dryden@vet.k-state.edu

${ }^{1}$ Department of Diagnostic Medicine/Pathobiology, Kansas State University,

Manhattan, KS 66506, USA

Full list of author information is available at the end of the article
} 


\section{Background}

Fleas are an extremely important blood sucking parasite that infest dogs and cats worldwide. Flea infestations are medically and economically important because fleas are not just irritating to dogs and cats, they can produce anemia, allergic dermatitis, carry several bacterial pathogens, and serve as the intermediate host for cestode and filarid parasites [1].

A number of field studies conducted in Australia, Europe and the United States have documented that a variety of modern topical and oral flea products can effectively eliminate flea infestations [2-14]. Compounds such as afoxolaner, dinotefuran-pyriproxyfen, fipronil $( \pm$, (s)-methoprene) imidacloprid, indoxacarb, fluralaner, lufenuron (+pyrethrin spray or + nitenpyram tablets), selamectin, and spinosad have been found in these various studies to be effective in reducing or eliminating flea infestations on naturally infested dogs and cats without the need for premises treatments [2-14].

Fluralaner and afoxolaner are recently introduced oral flea and tick adulticides in the isoxazoline class of drugs. Both drugs work as GABA-Chloride antagonists causing over excitation of the insect and arachnid nervous system and rapid ectoparasite death $[15,16]$. Both molecules have demonstrated rapid and persistent efficacy against fleas and multiple species of ticks $[17,18]$.

Following the administration of a fluralaner chew, efficacy has been maintained against fleas in both field and laboratory studies for 12 weeks $[14,19,20]$. A single dose of a fluralaner chew has been shown to start working within $2 \mathrm{~h}$ of administration, eliminating $88 \%$ of an existing flea population on dogs within $4 \mathrm{~h}$ [19]. In a separate study fluralaner killed newly acquired female fleas rapidly enough that no eggs were laid after repeated infestations for 120 days [21]. In multicentric field studies evaluating dogs not managed with associated medications, fluralaner's rapid and pronounced efficacy against fleas improved clinical signs associated with Flea Allergy Dermatitis (FAD) by 80-96\% [14] and $85.7 \%$ [20]. In those studies, the veterinarian's experience was used to determine if the signs were consistent with FAD. Recently, two more controlled studies using fluralaner were performed showing $98.8 \%$ and $100 \%$ resolution of FAD signs within 12 weeks, respectively in 20 dogs diagnosed with FAD $[22,23]$. In one study clinical signs of FAD were based upon clinical examination, and a positive response on serologic and intradermal evaluations of flea allergen [23]. The other study was an open pretreatment versus post-treatment assessment, where clinical signs of FAD were clinically evaluated by a veterinary dermatologist [22].

Afoxolaner has an onset of activity of $4 \mathrm{~h}$ against fleas [24] and has been shown to reduce flea egg production by greater than $99 \%$ for one month [25]. A laboratory study comparing one dose of fluralaner and 3 doses of afoxolaner administered 28 days apart showed a statistically significant difference in flea adulticide efficacy between products at 6 and $12 \mathrm{~h}$ post-infestation time points on days 70 and 84 . Throughout the 84 day study there was no statistically significant difference in flea reductions between products when assessed at $24 \mathrm{~h}$ after each weekly infestation with efficacies of $99.6 \%$ and $100 \%$ respectively on day 84 [26]. The flea reduction efficacy of afoxolaner administered monthly to client owned dogs in Tampa, FL was $100 \%$ within 6 weeks [11].

The objective of this study was to evaluate and compare the efficacy of fluralaner and afoxolaner oral treatments against fleas on naturally infested dogs in subtropical Florida using both premises and on-animal flea population estimating techniques. Additionally, this study was designed to determine the effect of these treatments on reducing flea associated dermatitis and pruritus. The methodology used in this investigation eliminated client compliance and adherence problems because investigators administered products to all animals.

\section{Methods}

\section{Home and pet study inclusion criteria}

Through referrals from Sunshine Animal Hospital, Tampa, FL, Animal Dermatology South, New Port Richey, FL, and advertisements on CRAIGSLIST $^{\oplus}, 36$ private residences were selected for inclusion in the study from May 19 - June 08, 2015.

Homes were selected based on the following criteria: 1) a minimum of five fleas observed in area flea counts on at least one dog at the residence; 2) a minimum of five fleas collected in a $16-24 \mathrm{~h}$ period in two intermittent light flea traps; 3) one to five healthy, non-fractious dogs at the residence (no cats); 4) qualifying dogs must spend $>50 \%$ of their time in the indoor premises; 5) homeowner's willingness to participate in the 3 month study; 6) owners agreeing not to use any other topical or premise flea control products during the study and no history of residual topical or oral flea products used in the previous 30 days; 7) owners agreeing not to bring any other mammalian pets into the household for the duration of the study; 8) no pregnant or nursing dogs in the household; 9) dogs qualifying for the study must be > 6 months of age and $>4.4 \mathrm{lb} ; 10$ ) completion of a questionnaire concerning pet habits, visiting pets, previous flea treatments and personal observations around their residence concerning wildlife and feral cats and 11) owners willingness to sign an informed consent form.

\section{Treatment groups}

Homes and dogs meeting these criteria were placed into 1 of 2 treatment groups. Home entry numbers (1-36) were each assigned a random number by Excel (Excel 
2013) and blocked into groups of 2 . The highest random number within each block was assigned to group 1 and lowest to group 2.

Dogs in treatment group 1 were administered an oral fluralaner chew (Bravecto ${ }^{\circ}$, Merck Animal Health) once on day 0 according to label dosing recommendations.

Dogs in group 2 were administered an oral afoxolaner chewable (NexGard ${ }^{\circ}$ Chewables, Merial) according to the label dosing recommendations 3 times during the $82-86$ day study: once on day 0 , once between days $28-$ 30 and once again between days 54-60.

All dogs were weighed prior to each treatment and products were administered by members of the Kansas State University (K-State) Flea Team who were not blinded to treatment groups. While only dogs meeting the inclusion criteria were included in the study for data collection, all dogs living at a residence were administered group appropriate treatment. No other on-animal or oral flea product or premises flea treatments were used during the 12 week study. There were no restrictions on the animals regarding exposure to rain, swimming, bathing, or movement outdoors.

This study was conducted without a placebo control group. While the use of a non-treated group might have provided a better evaluation of the performance of the two treatment regimens, it is the opinion of these authors that the large flea infestations commonly encountered in the Tampa, FL area preclude the use of a non-treated group. Withholding treatment would be detrimental to the health and welfare of both the dogs, and potentially even the humans in a household.

\section{Flea population assessment}

The numbers of adult fleas present in the indoor premises were assessed using intermittent light traps [3-8, 10 , $11,27,28]$. One trap was placed in each of two rooms for 16 to 24-h. Rooms were selected based on where the $\operatorname{dog}(\mathrm{s})$ spent most of the time or where owners had observed fleas. Once rooms were selected, the traps were returned to the same rooms in the same location at every counting period. Fleas collected on the adhesive pads of the traps were enumerated and identified by microscopic observation as to determine species.

The flea population on each pet was assessed using a visual area count methodology $[3-8,10,11,29]$. Area counts were performed at five locations on each animal; dorsal midline, tail head, left lateral, right lateral, and inguinal region. Area counts were limited to one minute per location and conducted by parting the hair against the lay using both hands until the area was covered. Maximum number of fleas per zone was capped at 50; therefore, the maximum total area flea counts for a dog was 250. Area (on-animal) and environment flea counts were conducted \pm 1 day on days $0,7,14,21$, then once between days 28-30, 40-45, 54-60 and 82-86. Personnel conducting pet and premises flea counts were not blinded to treatment groups.

\section{Evaluation of skin disease and pruritus}

At visits occurring on days $0,14,28-30,40-45,54-60$, and $82-86$, the owner was asked to rate the pruritus level of the qualifying $\operatorname{dog}(\mathrm{s})$ in the home on a nonnumeric scale using a data capture form. This form provides 6 written descriptions of increasing pruritus severity, from "Normal dog-I do not think itching is a problem" through "Extremely severe itching..." (Fig. 1) $[30,31]$. Following the rating of pruritus by the owner, a numeric scale of $0-10$ was placed on the form and a numeric assessment of the pruritus level was recorded. Owners did not see and were not informed of the numerical scale or their $\operatorname{dog}(\mathrm{s})$ score during the study. In a household with more than one owner, only one owner was allowed to assess the pruritus level of the $\operatorname{dog}(\mathrm{s})$ throughout the study.

Masked as to treatment groups, clinical dermatologic observations were made on days $0,30,60$, and 84 of the study ( \pm 3 days) of all qualifying dogs in the homes. To ensure repeatability of results, a single Board-certified veterinary dermatologist $(\mathrm{MC})$ made all of the observations. To assess skin lesions two different systems were used. Dermatologic signs potentially associated with Flea Allergy Dermatitis were assessed using a flea bite hypersensitivity severity scale that evaluated erythema, papules, crusts, scale, alopecia, and excoriation [32]. Each of these categories was graded by the boardcertified veterinary dermatologist using a scale from 0 to $3,0=$ no signs, $1=$ mild, $2=$ moderate, $3=$ severe Three body sites were assessed, (1) dorsum, from the withers to the base of the tail, (2) left and right lateral thorax, just caudal to the elbow and extending to the last rib, (3) "flea triangle", including the dorsal lumbosacral region, caudomedial thighs, and ventral abdomen (Table 1) [32]. Scores for each of the six dermatology categories, for each body site, and the total sum of all of these sites and conditions were calculated for each dog at each observation. Additionally, dogs were also assessed by the dermatologist using the canine atopic dermatitis (CAD) extent and severity index scoring system (CADESI-4) (Table 2) [33], as a means to assess the skin in regions more commonly thought to be abnormal in atopic dermatitis patients. There is, however, overlap in the two scoring systems.

\section{Data analysis}

The dog and trap counts were analyzed separately at each time point, using the two-sample $t$ test to test for differences in the two groups. Statistical analysis was performed using SAS version 9.1 or higher. The animal 


\begin{tabular}{|l|l|}
\hline & $\begin{array}{l}\text { Extremely severe itching/almost continuous } \\
\text { Itching doesn't stop whatever is happening, even in the } \\
\text { consulting room (needs to be physically restrained from itching) } \\
\text { Severe itching/prolonged episodes } \\
\text { Itching might occur at night (if observed) and also when eating, } \\
\text { playing, exercising or being distracted }\end{array}$ \\
$\begin{array}{l}\text { Moderate itching/regular episodes } \\
\text { Itching might occur at night (if observed) but not when eating, } \\
\text { playing, exercising or being distracted }\end{array}$ \\
$\begin{array}{l}\text { Mild itching/a bit more frequent } \\
\text { Wouldn't itch when sleeping, eating, playing, exercising or } \\
\text { being distracted }\end{array}$ \\
$\begin{array}{l}\text { Very mild itching/only occasional episodes } \\
\text { The dog is slightly more itchy than he/she was before the skin } \\
\text { problem started }\end{array}$ \\
Formal dog - I don't think itching is a problem \\
Pruritus Visual Analogue Scale
\end{tabular}

and trap flea count data were transformed prior to analysis using the $\mathrm{Y}=\log (\mathrm{x}+1)$ transformation.

The log transformed flea counts on dogs were analyzed by a mixed linear model with repeated measure including treatment, day, treatment by day as the fixed effects; and household, and animal as random effects. The log transformed flea counts in traps were analyzed by a mixed linear model with repeated measure including treatment,

Table 1 Flea bite hypersensitivity severity scoring form

\begin{tabular}{|c|c|c|c|c|}
\hline & $\begin{array}{l}\text { Site } 1 \\
\text { (Dorsum) }\end{array}$ & $\begin{array}{l}\text { Site } 2 \\
\text { (Lateral Thorax) }\end{array}$ & $\begin{array}{l}\text { Site } 3 \\
\text { (Flea Triangle) }\end{array}$ & $\begin{array}{l}\text { Sum of all sites } \\
\text { and categories }\end{array}$ \\
\hline \multicolumn{5}{|l|}{ Erythema } \\
\hline \multicolumn{5}{|l|}{ Papules } \\
\hline \multicolumn{5}{|l|}{ Crusts } \\
\hline \multicolumn{5}{|l|}{ Scale } \\
\hline \multicolumn{5}{|l|}{ Alopecia } \\
\hline \multicolumn{5}{|l|}{ Excoriation } \\
\hline \multicolumn{5}{|l|}{ Total scores } \\
\hline $\begin{array}{l}\text { Scale: } \\
0 \text { - no signs } \\
1 \text { - mild } \\
2 \text { - moderate } \\
3 \text { - severe }\end{array}$ & & & & \\
\hline
\end{tabular}

day, and treatment by day as the fixed effects and household as random effect.

A Kenward-Rogers adjustment was used to determine the denominator degree of freedom for hypothesis. Akaike's Information Criterion (AIC) was used as the criterion to select the covariance structure for repeated measures.

The dermatology, pruritus and CADESI scores were analyzed by the same mixed linear model with repeated measure as that for the flea counts on dogs.

Percentages of animals without fleas and the percentages of homes without fleas (defined as homes where no fleas were recovered on either traps or dogs), were analyzed and compared.

All comparisons were made between treatment groups on each data collection day and also between each collection day and the baseline (Day 0) values within each treatment group.

A two-tailed significance test was used for the comparison and significance was declared when $P<0.05$ and $90 \%$ confidence intervals were constructed for the differences between treatment groups for the equivalence declaration.

The primary software was SAS version 9.3 (SAS Institute Inc., Cary, NC, USA). 
Table 2 Canine Atopic Dermatitis Extent and Severity Index-4 (CADESI-4) scoring form

\begin{tabular}{|c|c|c|c|c|c|c|}
\hline \multirow{2}{*}{\multicolumn{2}{|c|}{ Perilabial Area (left and right combined) }} & \multirow[b]{2}{*}{1} & \multirow[t]{2}{*}{ Erythema } & \multirow[t]{2}{*}{ Lichenification } & \multirow[t]{2}{*}{ Excoriations and/or Alopecia } & \multirow[t]{2}{*}{ Total } \\
\hline & & & & & & \\
\hline \multirow{2}{*}{$\begin{array}{l}\text { Medial Pinnae } \\
\text { (concave pinnae) }\end{array}$} & Left & 2 & & & & \\
\hline & Right & 3 & & & & \\
\hline \multirow[t]{2}{*}{ Axillae } & Left & 4 & & & & \\
\hline & Right & 5 & & & & \\
\hline \multirow{2}{*}{$\begin{array}{c}\text { Front Paws } \\
\text { (dorsal and palmar sides combined) }\end{array}$} & Left & 6 & & & & \\
\hline & Right & 7 & & & & \\
\hline \multirow{2}{*}{$\begin{array}{c}\text { Hind Paws } \\
\text { (dorsal and palmar sides combined) }\end{array}$} & Left & 8 & & & & \\
\hline & Right & 9 & & & & \\
\hline \multirow{2}{*}{$\begin{array}{l}\text { Cubital Flexor } \\
\text { (elbow folds) }\end{array}$} & Left & 10 & & & & \\
\hline & Right & 11 & & & & \\
\hline \multirow{2}{*}{$\begin{array}{c}\text { Palmar Metacarpal } \\
\text { (from carpal to metacarpal pads) }\end{array}$} & Left & 12 & & & & \\
\hline & Right & 13 & & & & \\
\hline \multirow[t]{2}{*}{ Flanks } & Left & 14 & & & & \\
\hline & Right & 15 & & & & \\
\hline \multirow{2}{*}{$\begin{array}{l}\text { Inguinal Areas } \\
\text { (groin) }\end{array}$} & Left & 16 & & & & \\
\hline & Right & 17 & & & & \\
\hline \multicolumn{2}{|l|}{ Abdomen } & 18 & & & & \\
\hline \multicolumn{2}{|c|}{$\begin{array}{c}\text { Perineum } \\
\text { (from vulva/scrotum to anus) }\end{array}$} & 19 & & & & \\
\hline \multicolumn{2}{|l|}{$\begin{array}{l}\text { Ventral Tail } \\
\text { (proximal) }\end{array}$} & 20 & & & & \\
\hline \multicolumn{2}{|c|}{$\begin{array}{l}\text { Grade each site and each lesion type: } \\
\text { None: } 0 \text {; mild: } 1 \text {; moderate: } 2 \text {; severe: } 3 \text {; }\end{array}$} & & & & Total score $(20 \times 3 \times 3=180)$ & \\
\hline
\end{tabular}

Percent control of fleas counts were calculated using geometric means with Abbott's formula:

Efficacy $(\%)=100 \times(\mathrm{MB}-\mathrm{MC}) / \mathrm{MB}$

Where:

- $\mathrm{MC}$ is the geometric mean number of fleas on flea count day

- $\mathrm{MB}$ is the geometric mean number of live fleas count on baseline.

Note: Efficacy was calculated using both geometric and arithmetic means; however, geometric means were considered as the primary approach for effectiveness evaluation.

\section{Results}

Thirty-six homes were originally enrolled in the study. However, two households (one in the fluralaner group and one in the afoxolaner group) did not remain in the study for at least 30 days; data from those households were not included. In the fluralaner group, the owner was not reliable in scheduling rechecks following initial treatment. The owner of the afoxolaner household brought puppies under 6 months of age into the home within three weeks of treatment. Several other homes and/or dogs did not complete the entire 12-week study but remained up to days 40-45. Data from those homes and dogs were included in data analysis up to the point that follow up was no longer possible. In the fluralaner group, one home (1 dog) was lost after the day 40-45 recheck because the owner was unreachable by phone and was not found at the residence. In the afoxolaner group one dog was lost prior to the day 54-60 after succumbing to apparent "heat stroke". The other on study dog in the home completed the study. A home with two dogs treated with afoxolaner was lost on the last day of the study because the owner failed to appear for the appointment. A fourth afoxolaner dog was lost just prior to the last recheck when the owner moved out of the range of the study.

In the 17 homes in the fluralaner group that remained in the study beyond days 40-45 there were 34 dogs (avg. $15.1 \mathrm{~kg}$; range $2.0-36.0 \mathrm{~kg}$ ) enrolled. On day 0 dogs were treated in accordance with label direction and received a mean oral dose of $38.9 \mathrm{mg} / \mathrm{kg}$ (range $27.2-56.3 \mathrm{mg} / \mathrm{kg}$ ) fluralaner. There were an additional 9 dogs in these homes 
that did not qualify for the study because they had an insufficient numbers of fleas $(<5)$ on day 0 , resided permanently outdoors, were brought into the home after the enrollment date, or could not be safely handled by flea team members during flea counts. Therefore, there were a total of 43 dogs resident in the 17 homes that were treated with fluralaner on day 0 .

In the 17 homes in the afoxolaner group that remained in the study beyond days $40-45$, there were 27 dogs (avg. $16.7 \mathrm{~kg}$; range $3.6-36.8 \mathrm{~kg}$ ) enrolled in the study. On day 0 the dogs were treated in accordance with label direction and received a mean dose of $3.6 \mathrm{mg} / \mathrm{kg}$ (range $2.7-5.4 \mathrm{mg} / \mathrm{kg}$ ) afoxolaner. Dogs in the afoxolaner group were similarly treated by study personnel two additional times; on day 28-30 and day 54-60. There were an additional 9 dogs in these homes that did not qualify for the study because of the same factors listed previously. Therefore, there were a total of 36 dogs resident in the 17 homes that were treated with afoxolaner on day 0 .

On day 0 , pets in the fluralaner treatment group had a geometric mean of 28.3 (range 5-155) fleas observed in area counts (Table 3). Pets in the afoxolaner treatment group had a geometric mean of 20.4 (range 5-178) fleas observed in area counts on day 0 . The geometric mean flea count of fluralaner treated dogs was significantly higher on Day 0 than that of afoxolaner treated dogs $(P=0.004)$ Within 7 days of administration of fluralaner or afoxolaner the flea counts were reduced by $99.00 \%$ and $99.3 \%$, respectively (Table 3 ). By days $28-30$ the flea counts in the fluralaner treatment group were reduced by $100.0 \%$ and reductions remained at $99.9 \%$ to $100 \%$ for the remainder of the 12-week study following a single oral dose on day 0 (Table 3). Afoxolaner also markedly reduced flea populations, with $99.9 \%$ control on day $28-30$ and $99.9 \%-100 \%$ control out to days 82-86 after 3 monthly doses. (Table 3 ). Both products significantly reduced flea counts on dogs at each time point $(P<0.001)$. The flea counts of the two treatment groups were not significantly different on any of the post-treatment count days $(P \geq 0.327)$.

The number of flea free dogs in both groups following treatment were similar (Table 3). Following a single oral dose of fluralaner $88.2 \%$ (30/34) of dogs were completely flea free by day 14 and only a single flea was found on one dog between days $28-30$ and days $82-86$, with all dogs flea free at the end of the study. Similarly $81.5 \%(22 / 27)$ of the dogs in the afoxolaner treatment group were flea free by day 14 and only a single flea was found at two time points between days 28-30 and 82-86 following three monthly doses. All dogs were flea free by the end of the study (Table 3). Again, both products produced statistically significant more flea free dogs at each time point relative to study onset, $(P<0.001)$ but there was no statistically significant difference in the number of flea free dogs between treatment groups $(P \geq 0.4407)$.

During the entire 12-week study, 1,267 fleas were trapped in the 34 residences using intermittent light traps and all were identified as Ctenocephalides felis felis, the cat flea. On day 0 , the traps collected a geometric mean of 19.2 (range 6-122) and 14.0 (range 6-167) fleas in homes in the fluralaner and afoxolaner treatment groups, respectively (Table 4). Reductions in premises flea trap counts were $99.6 \%$ and $100.0 \%$ by days $28-30$ and $82-86$, respectively in the homes where pets were treated with fluralaner (Table 4). Reductions in premises flea trap counts were similar in homes where pets were treated with afoxolaner, with $96.7 \%$ and $98.9 \%$ by days $28-30$ and $82-86$, respectively (Table 4 ).

Households without fleas on pets or in traps were considered flea free. Treatment with one dose of fluralaner resulted in $88.2 \%(15 / 17)$ flea free households

Table 3 On-animal flea counts in naturally infested homes when dogs were administered either fluralaner or afoxolaner oral treatments

\begin{tabular}{|c|c|c|c|c|c|c|c|c|c|c|}
\hline \multirow{2}{*}{$\begin{array}{l}\text { Treatment } \\
\text { group }\end{array}$} & \multirow{2}{*}{$\begin{array}{l}\text { \# dogs at } \\
\text { day } 0\end{array}$} & & \multicolumn{8}{|c|}{ Days post-Treatment ${ }^{a}$} \\
\hline & & & Day 0 & Day 7 & Day 14 & Day 21 & Day 28-30 & Day 40-45 & Day 54-60 & Day $82-86$ \\
\hline \multirow[t]{4}{*}{ Fluralaner $^{\mathrm{b}}$} & 34 & Geomean $^{d}$ & 28.3 & 0.3 & 0.1 & 0.1 & 0.0 & 0.0 & 0.0 & 0.0 \\
\hline & & range & (5-155) & $(0-6)$ & $(0-3)$ & $(0-2)$ & $(0-0)$ & $(0-1)$ & $(0-0)$ & $(0-0)$ \\
\hline & & $\%$ control $^{\mathrm{e}}$ & & 99.0 & 99.6 & 99.8 & 100.0 & 99.9 & 100.0 & 100.0 \\
\hline & & $\begin{array}{l}\% \text { (\#) dogs with } \\
\text { no fleas }\end{array}$ & $\begin{array}{l}0.0 \\
(0 / 34)\end{array}$ & $\begin{array}{l}76.5 \\
(26 / 34)\end{array}$ & $\begin{array}{l}88.2 \\
(30 / 34)\end{array}$ & $\begin{array}{l}94.1 \\
(32 / 34)\end{array}$ & $\begin{array}{l}100.0 \\
(34 / 34)\end{array}$ & $\begin{array}{l}97.1 \\
(33 / 34)\end{array}$ & $\begin{array}{l}100.0 \\
(33 / 33)\end{array}$ & $\begin{array}{l}100.0 \\
(33 / 33)\end{array}$ \\
\hline \multirow[t]{4}{*}{ Afoxolaner ${ }^{c}$} & 27 & Geomean & 20.4 & 0.1 & 0.2 & 0.1 & 0.0 & 0.0 & 0.0 & 0.0 \\
\hline & & range & (5-178) & $(0-4)$ & $(0-2)$ & $(0-3)$ & $(0-1)$ & $(0-0)$ & $(0-1)$ & $(0-0)$ \\
\hline & & $\%$ control & & 99.3 & 99.2 & 99.6 & 99.9 & 100.0 & 99.9 & 100.0 \\
\hline & & $\begin{array}{l}\% \text { (\#) dogs with } \\
\text { no fleas }\end{array}$ & $\begin{array}{l}0.0 \\
(0 / 27)\end{array}$ & $\begin{array}{l}85.2 \\
(23 / 27)\end{array}$ & $\begin{array}{l}81.5 \\
(22 / 27)\end{array}$ & $\begin{array}{l}92.6 \\
(25 / 27)\end{array}$ & $\begin{array}{l}96.3 \\
(26 / 27)\end{array}$ & $\begin{array}{l}100.0 \\
(27 / 27)\end{array}$ & $\begin{array}{l}96.2 \\
(25 / 26)\end{array}$ & $\begin{array}{l}100.0 \\
(23 / 23)\end{array}$ \\
\hline
\end{tabular}

${ }^{a}$ In both groups, dogs were treated on day 0 . In the afoxolaner group, dogs were also treated once between days 28-30 and once between days $54-60$

${ }^{b}$ Dogs were orally administered fluralaner chews (Bravecto ${ }^{\oplus}$ Merck Animal Health) according to label directions

${ }^{c}$ Dogs were orally administered afoxolaner chewables (NexGard ${ }^{\circledR}$ Merial, Inc.) according to label directions

${ }^{\mathrm{d}}$ Geometric mean numbers of fleas in visual area counts on pets

$\mathrm{e}_{\{(\text {Day } 0}$ geometric mean animal area flea counts-day $\mathrm{x}$ geometric mean animal area flea counts)/day 0 geometric mean animal area flea counts $\}$ $\times 100$ 
Table 4 Fleas recovered in premises flea traps in naturally infested homes when dogs were administered either fluralaner or afoxolaner chews

\begin{tabular}{|c|c|c|c|c|c|c|c|c|c|c|}
\hline \multirow[t]{2}{*}{ Treatment group } & \multirow[t]{2}{*}{ \# homes } & & \multicolumn{8}{|c|}{ Days post-Treatment ${ }^{a}$} \\
\hline & & & Day 0 & Day 7 & Day 14 & Day 21 & Day 28-30 & Day $40-45$ & Day 54-60 & Day $82-86$ \\
\hline \multirow[t]{3}{*}{ Fluralaner $^{\mathrm{b}}$} & 17 & Geomean $^{d}$ & 19.2 & 1.5 & 0.5 & 1.2 & 0.1 & 0.1 & 0.0 & 0.0 \\
\hline & & $\%$ control $^{e}$ & & 92.4 & 97.3 & 93.9 & 99.6 & 99.7 & 99.8 & 100.0 \\
\hline & & range & $(6-122)$ & $(0-8)$ & $(0-5)$ & $(0-4)$ & $(0-1)$ & $(0-2)$ & $(0-1)$ & $(0-0)$ \\
\hline \multirow[t]{3}{*}{ Afoxolaner ${ }^{c}$} & 17 & Geomean & 14.0 & 1.9 & 1.7 & 1.4 & 0.5 & 0.3 & 0.3 & 0.1 \\
\hline & & $\%$ control & & 86.5 & 88.0 & 90.2 & 96.7 & 98.2 & 97.8 & 98.9 \\
\hline & & range & $(6-167)$ & $(0-10)$ & $(0-12)$ & $(0-23)$ & $(0-26)$ & $(0-11)$ & $(0-8)$ & $(0-1)$ \\
\hline
\end{tabular}

${ }^{a}$ In both groups, dogs were treated on day 0 . In the afoxolaner group dogs were also treated once between days 28-30 and once between days $54-60$

${ }^{b}$ Dogs were orally administered fluralaner chews (Bravecto ${ }^{\oplus}$ Merck Animal Health) according to label directions

${ }^{\circ}$ Dogs were orally administered afoxolaner chewables (NexGard ${ }^{\circledR}$ Merial, Inc.) according to label directions

${ }^{\mathrm{d}}$ Geometric mean numbers of fleas recovered in two intermittent light flea traps averaged within households

$\mathrm{e}_{\{(\text {Day } 0} 0$ geometric mean trap flea counts-day $\mathrm{x}$ geometric mean trap flea counts)/day 0 geometric mean trap flea counts $\left.)\right\} \times 100$

within 28-30 days of treatment and $100 \%$ of households (16/16) free of fleas at week 12. Treatment with three monthly doses of afoxolaner resulted in $70.6 \%(12 / 17)$ flea free households by day $28-30$ and $80 \%(12 / 15)$ of households free of fleas at week 12. There was no statistically significant difference in the number of flea free households between treatment groups $(P \geq 0.1012)$.

Dogs in the fluralaner and afoxolaner treatment groups had mean total flea bite hypersensitivity severity lesion scores of 12.8 (range 0-31) and 10.7 (range 1-30), respectively (Table 5 ) on day 0 . By the end of the three-

Table 5 Assessment of skin lesions using a flea bite hypersensitivity severity scale for dogs naturally infested with fleas and administered either fluralaner or afoxolaner oral treatments

\begin{tabular}{|c|c|c|c|c|c|}
\hline \multirow{2}{*}{$\begin{array}{l}\text { Treatment } \\
\text { group }\end{array}$} & & \multicolumn{4}{|c|}{ Days post-treatment ${ }^{a}$} \\
\hline & & Day 0 & Day $28-30$ & Day $54-60$ & Day $82-86$ \\
\hline \multirow[t]{5}{*}{ Fluralaner $^{b}$} & \# Dogs & 34 & 34 & 33 & 33 \\
\hline & Mean Score ${ }^{d}$ & 12.8 & 5.4 & 3.7 & 1.8 \\
\hline & STDEV & 8.22 & 4.13 & 3.80 & 1.64 \\
\hline & Range & $(0-31)$ & $(0-21)$ & $(0-21)$ & $(0-7)$ \\
\hline & Reduction $^{\mathrm{e}}$ & & $58.1 \%$ & $71.0 \%$ & $85.5 \%$ \\
\hline \multirow[t]{5}{*}{ Afoxolaner $^{c}$} & \# Dogs & 27 & 27 & 26 & 23 \\
\hline & Mean Score ${ }^{d}$ & 10.7 & 4.9 & 2.7 & 2.0 \\
\hline & STDEV & 7.15 & 3.35 & 2.76 & 2.30 \\
\hline & Range & $(1-30)$ & $(0-12)$ & $(0-13)$ & $(0-8)$ \\
\hline & Reduction & & $54.0 \%$ & $74.5 \%$ & $81.3 \%$ \\
\hline
\end{tabular}

an both groups dogs were treated on day 0 . In the afoxolaner group dogs were also treated once between days 28-30 and once between days 54-60 ${ }^{b}$ Dogs were orally administered fluralaner chews (Bravecto ${ }^{\oplus}$ Merck Animal Health) according to label directions

'Dogs were orally administered afoxolaner chewables (NexGard ${ }^{\circledR}$ Merial, Inc.) according to label directions

${ }^{\mathrm{d}}$ Arithmetic mean Flea Allergy Dermatitis lesion score (Wilkerson et al. Vet Immunol Immunopathol 99(3-4):179-192, 2004)

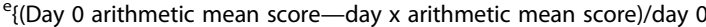
arithmetic mean score) $\} \times 100$ month study flea bite hypersensitivity severity lesion scores in the dogs were reduced to 1.8 (85.5\% improvement) and 2.0 (81.3\% improvement), in the fluralaner and afoxolaner treatment groups respectively (Table 5 ). Both products produced statistically significant reductions in signs of flea bite hypersensitivity relative to study onset, $(P<0.001)$, but there was no statistically significant difference between treatment groups $(P \geq 0.087)$. Interestingly, CADESI-4 lesion scores were also similarly reduced. On day 0 , dogs in the fluralaner and afoxolaner treatment groups had mean total CADESI-4 lesion scores of 51.5 (range 9-80) and 51.7 (range 3-91), respectively (Table 6). By the end of the three-month study CADESI-4 lesion scores in the dogs were reduced to 9.1 (82.3\% improvement) in the fluralaner treatment group and 8.7 (83.2 \% improvement), in the afoxolaner treatment group (Table 6). Both products produced statistically significant reductions in CADESI-4 lesion scores relative to study onset, $(P<0.001)$, but there was no statistically significant difference between treatment groups ( $P \geq 0.493)$.

Owner assessed pruritus scores showed rapid and marked improvement in both treatment groups. On day 0 , dogs in the fluralaner and afoxolaner treatment groups had mean pruritus visual analogue scale (PVAS) scores of 7.3 (range 2.2-10) and 7.1 (range 2.7-10), respectively (Table 6). Within 2 weeks post-treatment, mean pruritus scores had dropped by $71.0 \%$ (2.1) and $73.8 \%$ (1.9) in the fluralaner and afoxolaner treatment groups, respectively (Table 7). By the end of the study the mean pruritus scores in both groups had fallen to 0.9 and 0.7 , in the fluralaner and afoxolaner treatment groups, respectively (Table 7). Both products produced statistically significant reductions in PVAS scores relative to study onset, $(P<0.001)$, but there was no statistically significant difference between treatment groups $(P \geq 0.695)$.

Nine adverse events were reported in treated dogs in both groups during the course of the trial. In the 
Table 6 Assessment of skin lesions using the Canine Atopic Dermatitis Extent and Severity Index-4 (CADESI-4) scale for dogs naturally infested with fleas and administered either fluralaner or afoxolaner oral treatments

\begin{tabular}{|c|c|c|c|c|c|}
\hline \multirow[t]{2}{*}{ Treatment group } & & \multicolumn{4}{|c|}{ Days post-Treatment ${ }^{\mathrm{a}}$} \\
\hline & & Day 0 & Day $28-30$ & Day 54-60 & Day $82-86$ \\
\hline \multirow[t]{5}{*}{ Fluralaner $^{\mathrm{b}}$} & \# Dogs & 34 & 34 & 33 & 33 \\
\hline & Mean CADESI - 4 Score $^{d}$ & 51.5 & 20.4 & 15.2 & 9.1 \\
\hline & STDEV & 17.53 & 8.99 & 9.95 & 5.29 \\
\hline & Range & $(9-80)$ & $(0-39)$ & $(0-40)$ & $(0-20)$ \\
\hline & Reduction ${ }^{e}$ & & $60.4 \%$ & $70.5 \%$ & $82.3 \%$ \\
\hline \multirow[t]{5}{*}{ Afoxolaner ${ }^{c}$} & \# Dogs & 27 & 27 & 26 & 23 \\
\hline & Mean CADESI - 4 Score $^{d}$ & 51.7 & 18.8 & 12.7 & 8.7 \\
\hline & STDEV & 22.28 & 14.80 & 12.51 & 7.70 \\
\hline & Range & $(3-91)$ & $(3-51)$ & $(0-48)$ & $(0-26)$ \\
\hline & Reduction ${ }^{\mathrm{e}}$ & & $63.6 \%$ & $75.4 \%$ & $83.2 \%$ \\
\hline
\end{tabular}

${ }^{\mathrm{a}}$ In both groups dogs were treated on day 0 . In the afoxolaner group, dogs were also treated once between days 28-30 and once between days $54-60$

${ }^{b}$ Dogs were orally administered fluralaner chews (Bravecto ${ }^{\circledR}$ Merck Animal Health) according to label directions

${ }^{\circ}$ Dogs were orally administered afoxolaner chewables (NexGard ${ }^{\circledR}$ Merial, Inc.) according to label directions

${ }^{d}$ Arithmetic mean Canine Atopic Dermatitis Extent and Severity Index (CADESI)-4 scores (Olivry et al. Vet Dermatol 25(2):77-85, 2014)

$\mathrm{e}_{\{(\text {Day }} 0$ arithmetic mean CADESI-4 score - day $\mathrm{x}$ arithmetic mean CADESI-4 score)/day 0 arithmetic mean CADESI- 4 score) $\}$ x 100

fluralaner treatment group, one owner reported that a dog was lethargic for 1 to 2 days following fluralaner administration, and was acting normally on the $3^{\text {rd }}$ day. Another owner reported that a fluralaner treated dog was lethargic and had increased pruritus for a few days during the week following treatment, and was acting normally before the 7 day recheck. Another owner reported that 27 days after fluralaner treatment, his 2 dogs ate grass and vomited. Another owner reported that 2 of the 5 fluralaner treated dogs in the home had periodic episodes of coughing during the study. This owner declined offer of diagnostic evaluation of the two dogs.
In the afoxolaner treatment group an owner reported that their dog vomited 27 days after treatment, and appeared normal and healthy at retreatment on day 29 . Another owner reported loose stools in an afoxolaner treated dog from day 9 through day 13. One owner reported that one of five afoxolaner treated dogs in the household died prior to the day 54-60 evaluation. The owner assessed the cause as "heat stroke" when the airconditioner broke during the day while the owner was absent. The owner disposed of the dog's body prior to informing the study investigators, and it was not possible to conduct a necropsy.

Table 7 Owner assessment of pruritus using a visual analogue scale (PVAS) for dogs naturally infested with fleas and administered either fluralaner or afoxolaner treatments

\begin{tabular}{|c|c|c|c|c|c|}
\hline \multirow{2}{*}{$\begin{array}{l}\text { Treatment } \\
\text { group }\end{array}$} & & \multicolumn{4}{|c|}{ Days post-Treatment ${ }^{\mathrm{a}}$} \\
\hline & & Day 0 & Day 28-30 & Day 54-60 & Day $82-86$ \\
\hline \multirow[t]{5}{*}{ Fluralaner $^{b}$} & \# Dogs & 34 & 34 & 33 & 33 \\
\hline & Mean PVAS Score & 7.3 & 1.7 & 0.9 & 0.9 \\
\hline & STDEV & 2.40 & 1.8 & 1.2 & 1.3 \\
\hline & Range & $(2.2-10)$ & $(0-6)$ & $(0-4.2)$ & $(0-4.2)$ \\
\hline & Reduction ${ }^{e}$ & & $77.0 \%$ & $87.5 \%$ & $88.0 \%$ \\
\hline \multirow[t]{5}{*}{ Afoxolaner ${ }^{c}$} & \# Dogs & 27 & 27 & 26 & 23 \\
\hline & Mean PVAS Score ${ }^{d}$ & 7.1 & 1.5 & 0.7 & 0.7 \\
\hline & STDEV & 2.2 & 1.8 & 0.9 & 1.4 \\
\hline & Range & $(2.7-10)$ & $(0-6.5)$ & $(0-3.6)$ & $(0-6.7)$ \\
\hline & Reduction & & $79.2 \%$ & $89.6 \%$ & $89.7 \%$ \\
\hline
\end{tabular}

${ }^{\mathrm{a}}$ In both groups dogs were treated on day 0 . In the afoxolaner group dogs were also treated once between days 28-30 and once between days $54-60$

${ }^{b}$ Dogs were orally administered fluralaner chews (Bravecto ${ }^{\oplus}$ Merck Animal Health) according to label directions

${ }^{c}$ Dogs were orally administered afoxolaner chewables (NexGard ${ }^{\circledR}$ Merial, Inc.) according to label directions

${ }^{\mathrm{d}}$ Arithmetic mean pruritus score as assessed by dog owners using the PVAS (Hill et al. Vet Dermatol 18(5):301-308, 2004)

$\mathrm{e}_{\{(\text {Day } 0}$ arithmetic mean FAD score-day $\mathrm{x}$ arithmetic mean FAD score)/day 0 arithmetic mean FAD score $\left.)\right\} \times 100$ 


\section{Discussion}

Fluralaner chews provided excellent flea control achieving $99.0 \%$ reduction in geometric mean area flea counts on dogs within one week and complete elimination of on-animal flea burdens within 2 months after a single dosage. There were still no fleas on any dog and none in any home at the end of the 12-week study. The residual activity of this formulation was remarkable given the potential for continual flea infestation pressure from infested indoor and outdoor premises. The afoxolaner chewables administered monthly also provided good flea control. Following three monthly oral doses of afoxolaner the geometric mean area flea counts on dogs was also reduced by $100 \%$ by the end of the 12 week study and $>80 \%$ of homes were flea free. The level of efficacy observed in this study for the afoxolaner chewable was very similar to that observed in a previous field study conducted in west central Florida (Tampa Bay) [11].

The area count technique used in this and previous in-home investigations in west central Florida has been shown to detect an average of $23.5 \%$ of the total pet flea burden [29]. Therefore, average pretreatment total body flea burdens of dogs in the fluralaner and afoxolaner treatment groups based on geometric means area counts of 28.3 and 20.4 can be estimated to be approximately 120 and 86.8 for dogs in this study, respectively.

It is important to note that in previous in-home studies conducted in west central Florida the percent reductions in on-animal flea counts were often less than $100 \%$ even through 90 days of treatment $[3-8,10]$. In a 2010 study where dogs were treated with either a dinotefuran-pyriproxyfen or fipronil (s)-methoprene topical spot-on formulation, only $60.0 \%$ and $55.6 \%$ of dogs were completely flea free by days 54-60 [8]. In 2013 even though the efficacy against on-animal flea burdens of monthly topical indoxacarb was $99.1 \%$, the number of completely flea free dogs was only $77.1 \%$, by the end of the two month study [10]. In that 2013 study, the monthly application of fipronil (s)-methoprene topical spot-on only achieved $15.6 \%$ flea free status by 2 months.

In contrast to these previous studies, when dogs were administered either fluralaner or afoxolaner oral treatments, $88.2 \%$ and $81.5 \%$ were flea free within 2 weeks and $100 \%$ and $96.3 \%$ were flea free within 4 weeks, respectively. These data combined with the on-animal flea count efficacy data are indicating that these isoxazolines have a very rapid residual speed of kill under natural flea exposure conditions. Previous reports from a laboratory study suggest minor differences in the speed of kill may have clinical consequences [26]. However, this real world field study demonstrated no statistically significant differences between one dose of fluralaner and three doses of afoxolaner in any of the flea infestation or clinical parameters assessed. In addition, the continued residual efficacy of a single oral dose of fluralaner was evidenced by the fact that from evaluations conducted between days 28-30 post-treatment up to and including evaluations on week 12 , only a single flea was ever found on a single dog. Furthermore, one administration of fluralaner per dog cleared all households completely of fleas in the 12 weeks of this study.

In this current study one of the objectives was to assess the effect of these treatments on reducing dermatitis and pruritus in treated dogs. Flea allergy dermatitis and atopic dermatitis are the most common allergic skin diseases in dogs and cats [34]. To evaluate flea allergy dermatitis we chose a flea bite hypersensitivity lesional scoring system that was previously published from a laboratory controlled FAD induction study [32]. Although multiple validated scoring systems for canine atopic dermatitis exist, currently, the International Committee on Allergic Diseases of Animals (ICADA) recommends CADESI-4, or, canine atopic dermatitis lesion index (CADLI) as the only two validated severity scales to score skin lesions of canine atopic dermatitis patients [33]. CADESI-4 was utilized in this study for a more thorough dermatologic lesion assessment. It is important to note that a definitive diagnosis of FAD or CAD was not attained for the patients enrolled in the study. The intent was to have a single investigator clinically evaluate each of the study participants utilizing the aforementioned scoring systems to determine the clinical relevance of flea control measures when additional treatments were not provided.

The owner reported pruritus assessment tool (PVAS) we used was previously validated and used in field trials $[9,30,31]$. Pruritus is a subjective parameter and is important in evaluating response to therapy in a patient with pruritic dermatitis [30, 31]. There was rapid statistically significant improvement in PVAS scores for patients receiving fluralaner or afoxolaner despite no concurrent therapies.

The diagnosis of flea allergy dermatitis is made based on history, clinical signs, and response to anti-flea therapy [35]. Intradermal allergy testing and serological testing can have variable results and are not recommended for diagnosis of flea allergy on their own. Specificity, sensitivity, and accuracy of intradermal testing with whole-body flea extracts can vary, especially if performed in a flea endemic region [35]. Definitive diagnosis of flea allergy dermatitis must be made by the resolution of clinical signs in response to flea control and the recurrence of those signs upon re-exposure to fleas. In this current study there was statistically significant improvement in the flea bite hypersensitivity severity scores in both treatment groups. By days 54-60, there was $85.5 \%$ and $81.3 \%$ improvement with fluralaner and afoxolaner, respectively. The marked improvement in PVAS as well as the FAD scores upon treatments with fluralaner and 
afoxolaner supports that most of the participants had clinical signs attributable to flea exposure.

Diagnosis of canine atopic dermatitis is mainly a diagnosis of exclusion that requires a systematic approach [36, 37]. Diagnosis is based on history, clinical signs, and ruling out other pruritic diseases that share similar characteristics [36, 37]. Many of the study participants had lesions consistent with those that can be seen in atopic dermatitis, however further evaluation would be necessary for this diagnosis to be made. Dogs exhibited clinical signs consistent with atopic dermatitis, though it was beyond this study to pursue a more definitive diagnosis. The improvement of CADESI-4 scores, could support the concept that there is a threshold for the development of atopic dermatitis. The concept of threshold for the development of atopic dermatitis relates to the allergen load. When the allergen load is low, no symptoms may be observed, however when a heavy allergen burden exists, clinical disease results [34]. In our study, the elimination of fleas without the benefit of additional treatments caused statistically significant improvement in PVAS, CADESI-4, and FAD scores. Therefore, it is possible that the elimination of fleas resulted in a reduction of stimuli below the pruritic threshold, and/or, that the allergen burden was reduced such that if participants were atopic dogs, they were able to experience significant improvement [34].

An adverse event is any clinical sign reported following treatment administration regardless of causality. Adverse events were reported following administration of both fluralaner and afoxolaner in the course of this study. The reported events in this study may have been treatment associated or may have been independent of treatment administration. All but one of the reports were considered to be not serious and all of these resolved without treatment, with none of the affected dogs withdrawn from the study. The afoxolaner treated dog that died was unfortunately not available for necropsy examination.

Despite study protocol requirements that dogs not be pregnant, breeding or lactating during the 84-day study, one dog in the fluralaner treated group did become pregnant and delivered puppies on study day 49. As fluralaner is approved for pregnant and lactating dogs, this dog continued in the study and birthed a healthy litter of puppies.

\section{Conclusions}

This clinical field investigation conducted during the summer of 2015 in subtropical west central Florida clearly demonstrated that these oral isoxazoline formulations rapidly and effectively controlled flea populations in homes and on dogs. While the afoxolaner chewable was administered three times at monthly intervals, fluralaner eliminated fleas on dogs and in the homes within 12 weeks following a single dose. Not only were flea populations controlled by these formulations, but marked improvement in dermatologic lesions and pruritus were also observed without the use of any concurrent medical intervention.

\begin{abstract}
Abbreviations
AIC, Akaike's information criterion; CAD, canine atopic dermatitis; CADESI, canine atopic dermatitis extent and severity index scoring system; CADLI, canine atopic dermatitis lesion index; FAD, flea allergy dermatitis; ICADA, international

committee on allergic diseases of Animals; PVAS, pruritus visual analogue scale
\end{abstract}

\section{Acknowledgements}

We thank Dr. James Barrie, Dr. Mark Twilla and all the staff at Sunshine Animal Hospital, Tampa FL and all the staff at Animal Dermatology South, New Port Richey, FL for their invaluable assistance in conducting this study.

\section{Funding}

This study was funded in part by a grant from Merck Animal Health (Madison, NJ).

\section{Availability of data and material}

Raw data from this study has not been shared at this time because we anticipate additional publications exploring some of the detailed and complex dermatologic results.

\section{Authors' contributions}

MWD was primary author of study design, served as primary study investigator and drafted the manuscript. VS and TB coordinated and supervised data collection and entry and revision of manuscript. KK, AS, LC, JCM and KMF were responsible for animal handling and collection of data and data entry. MC, KG and $C T$ were responsible for blinded dermatology assessments. $\mathrm{KH}$ assisted in design of study, monitoring of study and manuscript revision. LM and CR assisted in monitoring of study and manuscript revision. FS conducted the statistical analysis of the data. All authors reviewed and approved the final manuscript.

\section{Competing interests}

MWD has had research projects funded at Kansas State University by Merck Animal Health and Merial and has had lectures sponsored by Merck Animal Health and Merial, manufacturers of Bravecto ${ }^{\oplus}$ and Nexgard ${ }^{\oplus}$ Chewables that were evaluated in these investigations. MC has had lectures sponsored by Merck Animal Health and Merial, manufacturers of Bravecto ${ }^{\circledast}$ and Nexgard ${ }^{\circledast}$ Chewables that were evaluated in these investigations. KH, LM, CR and FS are currently employed by Merck Animal Health.

\section{Ethics approval and consent to participate}

The present research was approved and complied with the regulations set forth by the Kansas State University Institutional Animal Care and Use Committee. This research protocol IACUC \# 3558 approved 09 April 2015. Each pet owner was required to read and sign an informed consent form prior to enrolling their dogs into this field trial.

\section{Author details}

${ }^{1}$ Department of Diagnostic Medicine/Pathobiology, Kansas State University, Manhattan, KS 66506, USA. ${ }^{2}$ Animal Dermatology South, 7741 Congress St, New Port Richey, FL 34653, USA. ${ }^{3}$ Merck Animal Health, 2 Giralda Farms, Madison, NJ 07940, USA.

Received: 1 March 2016 Accepted: 16 June 2016

Published online: 28 June 2016

References

1. Blagburn BL, Dryden MW. Biology, treatment and control of flea and tick infestations. Vet Clin N Am. 2009;39(6):1173-200.

2. Beugnet F, Franc M. Results of a European multicentric field efficacy study of fipronil-(S) methoprene combination on flea infestation of dogs and cats during 2009 summer. Parasite. 2010;17(4):337-42.

3. Dryden MW, Perez HR, Ulitchny DM. Control of flea populations on naturally infested dogs and cats and in private residences with either topical imidacloprid spot application or the combination of oral lufenuron and pyrethrin spray. Am J Vet Med Assoc. 1999;215(1):36-9. 
4. Dryden MM, Magid-Denenberg T, Bunch S. Control of fleas on naturally infested dogs and cats and in private residences with topical spot applications of fipronil or imidacloprid. Vet Parasitol. 2000;93:69-75.

5. Dryden M, Maggid-Denenberg T, Bunch S, Schenker R. Control of fleas on dogs and cats and in private residences with the combination of oral lufenuron and nitenpyram. Vet Therapeutics. 2001;2:208-14.

6. Dryden MW, Burkindine S, Lewis T, Houdeshell L, Rodriquez I, Hack R. Efficacy of selamectin in controlling natural flea infestations on pets and in private residences in comparison with imidacloprid and fipronil. In: proceedings of the American Association of Veterinary Parasitologists. Boston: 2001. p. 57

7. Dryden M, Carithers D, McBride A, Riggs B, Smith L, Davenport J, Smith V, Payne P, Gross S. A comparison of flea control measurement methods for tracking flea populations in highly infested private residences in Tampa FL, following topical treatment of pets with FRONTLINE ${ }^{\oplus}$ Plus (fipronil/(S)methoprene). Intern J Appl Res Vet Med. 2011;9(4):356-567.

8. Dryden MW, Payne PA, Smith V, Riggs B, Davenport J, Kobuszewski D. Efficacy of dinotefuran-pyriproxyfen, dinotefuran-pyriproxyfen-permethrin and fipronil-(S)-methoprene topical spot-on formulations to control flea populations in naturally infested pets and private residences in Tampa. FL Vet Parasitol. 2011;182:281-6.

9. Dryden MW, Ryan WG, Bell M, Rumschlag AJ, Young LM, Snyder DE. Assessment of owner-administered monthly treatments with oral spinosad or topical spot-on fipronil/(S)-methoprene in controlling fleas and associated pruritus in dogs. Vet Parasitol. 2013;191:340-6.

10. Dryden MW, Payne PA, Smith V, Chwala M, Jones E, Davenport J, et al. Evaluation of indoxacarb and fipronil (s)-methoprene topical spot-on formulations to control flea populations in naturally infested dogs and cats in private residences in Tampa FL. USA. Parasit Vectors. 2013;6:366.

11. Dryden MW, Smith V, Chwala M, Jones E, Crevoiserat L, McGrady JC, et al. Evaluation of afoxolaner chewables to control flea populations in naturally infested dogs in private residences in Tampa FL, USA. Parasit Vectors. 2015;8:286.

12. Miller PF, Peters BA, Hort CA. Comparison of lufenuron and nitenpyram versus imidacloprid for integrated flea control. Vet Therapeutics. 2001;2(4):285-92.

13. Robertson-Plouch C, Baker KA, Hozak RR, Zimmermann AG, Parks SC, Herr C et al. Clinical field study of the safety and efficacy of spinosad chewable tablets for controlling fleas on dogs. Vet Therapeutics. 2008;9(1):26-36.

14. Meadows C, Guerino F, Sun F. A randomized, blinded, controlled USA field study to assess the use of fluralaner tablets in controlling canine flea infestations. Parasit Vectors. 2014;7:375.

15. Gassel M, Wolf $C$, Noack $S$, Williams $H$, llg T. The novel isoxazoline ectoparasiticide fluralaner: Selective inhibition of arthropod Y-aminobutyric acid- and L-glutamate-gated chloride channels and insecticidal/acaricidal activity. Insect Biochem Molec Biol. 2014;45:111-24.

16. Hartline EJ, Gould BR, Waddell ME, McDowell RG, Kinney JB, Lahm GP, et al. Discovery and mode of action of afoxolaner, a new isoxazoline parasiticide for dogs. Vet Parasit. 2014:201:179-89.

17. Freedom of Information Summary, NADA 141-406. Approved September 14,2013

18. Freedom of Information Summary, NADA 141-426. Approved May 15, 2014.

19. Taenzler J, Wengenmayer $\mathrm{C}$, Williams H, Fourie J, Zschiesche E, Roepke RKA, Heckeroth AR. Onset of activity of fluralaner (BRAVECTO ${ }^{\text {TM }}$ ) against Ctenocephalides felis on dogs. Parasit Vectors. 2014;7:567.

20. Rohdich N, Roepke RKA, Zschiesche E. A randomized, blinded, controlled and multi-centered field study comparing the efficacy and safety of Bravecto $^{\text {TM }}$ (fluralaner) against Frontline ${ }^{\text {TM }}$ (fipronil) in flea- and tick-infested dogs. Parasit Vectors. 2014;7:83.

21. Dryden MW, Smith V, Bennett T, Math L, Kallman J, Heaney K, Sun F. Efficacy of fluralaner flavored chews (Bravecto ${ }^{\circledR}$ ) administered to dogs against the adult cat flea, Ctenocephalides felis felis, and egg production. Parasit Vectors. 2015;8:364.

22. Crosaz O, Chapelle E, Cochet-Faivre N, Ka D, Hubinois C, Guillot J. Open field study on the efficacy of oral fluralaner for long-term control of flea allergy dermatitis in client-owned dogs in lle-de-France region. Parasit Vectors. 2016;9(1):174.

23. Fisara P, Shipstone M, von Berky A, von Berky J. A small-scale open-label study of the treatment of canine flea allergy dermatitis with fluralaner. Vet Dermatol. 2015;26(6):417-e98.
24. Kunkle BN, Drag MD, Chester TS, Larsen DL. Assessment of the onset of action of afoxolaner against existing adult flea (Ctenocephalides felis) infestations on dogs. Vet Parasitol. 2014;201:204-6.

25. Hunter III JS, Dumont P, Chester TS, Young DR, Fourie JJ, Larsen DL. Evaluation of the curative and preventative efficacy of a single oral administration of afoxolaner against cat flea Ctenocephalides felis infestations on dogs. Vet Parasitol. 2014;201:207-11.

26. Beugnet F, Liebenberg J, Halos L. Comparative speed of efficacy against Ctenocephalides felis of two oral treatments for dogs containing either afoxolaner or fluralaner. Vet Parasitol. 2015;207:297-301.

27. Dryden M, Broce A. Development of a flea trap for collecting newly emerged Ctenocephalides felis (Siphonaptera: Pulicidae) in homes. J Med Entomol. 1993;30:901-6.

28. Müller GC, Dryden MW, Revay EE, Kravchenko VD, Broce AC, Hampton K, et al. Understanding attraction stimuli of Ctenocephalides felis for non-chemical control methods. Med Vet Entomol. 2011;25(4):413-20.

29. Dryden M, Boyer J, Smith V. Techniques for estimating on animal populations of Ctenocephalides felis (Siphonaptera: Pulicidae). J Med Entomol. 1994;31:631-24.

30. Hill PB, Lau P, Rybnicek J. Development of an owner-assessed scale to measure the severity of pruritus in dogs. Vet Dermatol. 2007;18:301-8.

31. Rybnícek J, Lau-Gillard PJ, Harvey R, Hill PB. Further validation of a pruritus severity scale for use in dogs. Vet Dermatol. 2009;20:115-22.

32. Wilkerson MJ, Bagladi-Swanson M, Wheeler DW, Floyd-Hawkins K, Craig C, Lee KW, Dryden M. The immunopathogenesis of flea allergy dermatitis in dogs, an experimental study. Vet Immunol Immunopathol. 2004;99(3-4): 179-92.

33. Olivry T, Saridomichelakis M, Nuttall T, Bensignor E, Griffin CE, Hill PB. Validation of the Canine Atopic Dermatitis Extent and Severity Index (CADESI)-4, a simplified severity scale for assessing skin lesions of atopic dermatitis in dogs. Vet Dermatol. 2014;25(2):77-85.

34. Marsella R, Sousa CA. The ACVD task force on canine atopic dermatitis (XIII): threshold phenomenon and summation of effects. Vet Immunol Immunopathol. 2001;81(3-4):251-4.

35. Logas D. Diagnostic Investigation of Canine Flea Bite Allergy. In: Noli C, Foster A, Rosenkrantz W, editors. Veterinary Allergy. Oxford: John Wiley and Sons, Ltd; 2013. p. 149-51.

36. Miller W, Griffin C, Campbell K. Hypersensitivity Disorders. In: Muller and Kirk's Small Animal Dermatology. 7th ed. St Louis: Saunders-Elsevier; 2013. p. 372.

37. Griffin CE. Diagnosis of Canine Atopic Dermatitis. In: Noli C, Foster A, Rosenkrantz W, editors. Veterinary Allergy. Oxford: John Wiley and Sons, Ltd; 2013. p. 70-7.

\section{Submit your next manuscript to BioMed Central and we will help you at every step:}

- We accept pre-submission inquiries

- Our selector tool helps you to find the most relevant journal

- We provide round the clock customer support

- Convenient online submission

- Thorough peer review

- Inclusion in PubMed and all major indexing services

- Maximum visibility for your research

Submit your manuscript at www.biomedcentral.com/submit
Biomed Central 